\title{
Macrotrombocitopenia Como Marcador Para A Identificação De Indivíduos Com A Síndrome De Deleção 22q11 Entre Pacientes Com Cardiopatia Congênita
}

\author{
De Morais, C.O.; dos Santos, C.B.L.; Nogueira, L.T.; Yonamine, T.M.; \\ Fiegenbaum, M.; Rosa, R.F.M.; \\ Apresentador: Camila Ohomoto de Morais \\ Premiados - Menção Honrosa: Destaque acadêmico
}

Resumo

Introdução: a síndrome de deleção 22q11

(SD22q11), ou síndrome velocardiofacial/DiGeorge, é uma das causas conhecidas mais comuns de cardiopatia congênita (CC) e se caracteriza por um quadro clínico bastante variável, o que dificulta a sua identificação. Nosso objetivo foi avaliar se a macrotrombocitopenia poderia ser considerada um preditor diagnóstico para a SD22q11 entre pacientes com CC. Método: a amostra consistiu de uma coorte prospectiva e consecutiva de pacientes com CC hospitalizados pela primeira vez em uma unidade de tratamento intensivo cardíaca de um hospital pediátrico do Sul do país. Estes foram submetidos à avaliação através do cariótipo de alta resolução e à pesquisa de microdelação 22q11 pela técnica de hibridização in situ fluorescente (FISH), além da avaliação hematológica pela contagem e determinação do volume das plaquetas. Pacientes com anormalidades cromossômicas distintas da SD22q11 foram excluídos. Com o objetivo de verificar se a macrotrombocitopenia poderia ser um marcador para a identificação dos pacientes com a SD22q11, calculou-se a sensibilidade, a especificidade, o valor preditivo positivo (VPP), o valor preditivo negativo (VPN), a razão de verossimilhança positiva (RVP), a razão de verossimilhança negativa (RVN), a acurácia e a curva ROC para esta variável. Resultados: dos 198 pacientes avaliados através do cariótipo e do FISH, 28 apresentaram outras anormalidades cromossômicas, diferentes da SD22q11. Dos 170 pacientes restantes, em 13 não se obteve os resultados da avaliação hematológica das plaquetas. Assim, nossa amostra final foi composta de 157 pacientes. A mediana da contagem de plaquetas foi estatisticamente similar entre o grupo com $(226.000 / \mu \mathrm{L})$ e sem SD22q11 (324.000/ $\mu \mathrm{L})(\mathrm{P}=0,131)$. Macroplaquetas foram descritas em 4 pacientes $(2,5 \%)$, nenhum deles com a microdeleção 22q11. Trombocitopenia foi verificada em 11 (7\%), e um deles possuía a microdeleção 22q11 $(\mathrm{P}=0,251)$. Em relação à trombocitopenia como marcador diagnóstico para a SD22q11, a sensibilidade desta foi de $25 \%$, a especificidade de $93,4 \%$, o VPP de $9 \%$ e o VPN de $97,9 \%$. A RVP foi de 3,8, a RVN foi de 0,8 e a acurácia de $92 \%$. A área da curva ROC para esta variável foi de 0,408 ( $\mathrm{P}=0,529)$. Conclusões: apesar da macrotrombocitopenia ser cada vez mais considerada um achado do amplo espectro da SD22q11, esta pareceu ser um marcador pobre para a detecção da síndrome entre pacientes com CC.

\section{Referência:}

De Morais, C.O.; dos Santos, C.B.L.; Nogueira, L.T.; Yonamine, T.M.; Fiegenbaum, M.; Rosa, R.F.M.; Macrotrombocitopenia Como Marcador Para A Identificação De Indivíduos Com A Síndrome De Deleção 22q11 Entre Pacientes Com Cardiopatia Congênita. In: Il Congresso Brasileiro de Medicina Hospitalar - II CBMH [= Blucher Medical Proceedings, vol.1, num.5] São Paulo: Editora Blucher, 2014. p.4

DOI 10.5151/medpro-II-cbmh-013 